Proceedings of the 2000 ASME International Mechanical Engineering Congress and Exposition, Orlando, Florida (2000)

AMD-Vol. 241 pp. 119-123.

\title{
STABILITY OF HIGH-SPEED MILLING
}

\author{
Tamás Insperger \\ Budapest University of Technology and \\ Economics \\ Department of Applied Mechanics \\ Budapest, $\mathrm{H}-1521$, Hungary \\ inspi@galilei.mm.bme.hu
}

\author{
Gábor Stépán \\ Budapest University of Technology and \\ Economics \\ Department of Applied Mechanics \\ Budapest, $\mathrm{H}-1521$, Hungary \\ stepan@mm.bme.hu
}

\begin{abstract}
The stability charts of high-speed milling are constructed. Non-conventional unstable regions and vibration frequencies are identified. These are related to flip bifurcation, i.e. period doubling vibrations occur apart of the conventional self-excited vibrations typical for turning or low-speed milling with multiple active teeth. A new stability criterion is proposed and applied for the delayed parametric excitation model of milling.
\end{abstract}

\section{INTRODUCTION}

The introduction of the so-called regenerative effect resulted in a breakthrough in the modeling of machine tool chatter. This effect is related to the cutting force variation due to the wavy workpiece surface cut one revolution ago. After the extensive work of Tlusty et. al. (1962) and Tobias (1965), regenerative effect became the most commonly accepted explanation for machine tool chatter (see Moon, 1998). The corresponding mathematical models are delay-differential equations (DDE). General stability criteria for these equations in autonomous cases were given and applied for advanced cutting models of turning by Stépán $(1989,1998)$. The study of nonlinear phenomena during the turning process showed the existence of unstable periodic motions about the stable stationary cutting (a so-called subcritical Hopf bifurcation) which was shown experimentally by Shi and Tobias (1984) and analytically by Stépán and Kalmár-Nagy (1997).

Modeling of the milling process requires the description of a time-dependent resultant cutting force which varies with the number of active teeth. This may show a small periodic component only if this number is great. For these cases, the conventional time-averaging was used in the classical literature (see Tobias, 1965) and so the stability results were similar to those of turning. From mathematical view-point, this averaging can hardly be justified since significant errors (even qualitative ones) can occur due to the time-dependent parameters in the model (see Hale and Lunel, 1993, Farkas, 1994). A more precise model of milling leads to parametric excitation in the regenerative effect. The equation of motion is a DDE with periodic coefficients, and the stability criteria can not be given in closed form even in the linear case. Minis and Yanushevsky (1993) used the first harmonics of the time-periodic parameters and showed slight deviations in the stability of milling relative to the results with the time-averaging method. Budak and Altintas (1998a, 1998b) used $3^{\text {rd }}$ order harmonic balance in the milling problem. Seagalman and Butcher (2000) used harmonic balance method with much more harmonics and found greater deviations in some narrow parameter domains. The results were applied for turning where the system stiffness was varied periodically. The most sophisticated milling models of Balachandran and Zao (2000) were analyzed by extensive simulation, and also showed some deviations in the stability results.

Still, there are no reliable analytical results for the stability of linear time-dependent DDE models which could be used as reference for the analysis of milling dynamics. Clearly, the results of Davies et al. (2000) for highly interrupted cutting could take this role in the limit case of high speed milling with a single tooth cutting a thin workpiece. The models of highspeed milling with 2 or 3 active teeth require new methods which finally explain the transition between the two stability charts related to the two extreme models, i.e. the discrete map of highly interrupted cutting and the autonomous DDE of turning. The results explain the appearance of flip bifurcation in the milling process which is a qualitatively new phenomenon compared to the dynamics of turning. 


\section{MECHANICAL MODEL OF MILLING}

A 1 degree of freedom mechanical model of the milling process can be seen in Figure 1. The variational system around the stationary periodic motion of the milling tool can be written in the following form

$$
\ddot{x}(t)+2 \zeta \omega_{n} \dot{x}(t)+\omega_{n}{ }^{2} x(t)=\frac{1}{m} \Delta F_{x}(t),
$$

where $x(t)$ is the position of the tool edge at the time instant $t$, $\omega_{n}=\sqrt{k / m}$ is the natural angular frequency, $\zeta=c /\left(2 m \omega_{n}\right)$ is the relative damping factor and $m$ is the mass. $\Delta F_{x}(t)$ is the $x$ component of the cutting force variation, and because of the regenerative effect of cutting, it depends on the difference of the present and the delayed tool position

$$
\Delta F_{x}(t)=w h(t)(x(t-\tau)-x(t)) .
$$

Here, $w$ denotes the chip width, $h(t)$ is a periodic function with the tooth pass period $\tau$ according to the time varying number of the working teeth, and depends on the geometry of milling through the parameters tool diameter $D$, workpiece width $B$, difference $e$ between the centerlines of the tool and the workpiece, number $z$ of teeth.

In this paper we investigate the case of interrupted machining when the tool loses the contact with the workpiece for certain time intervals. In these time intervals, $h(t)$ is zero, while in case of contact, it takes (approximately) a constant value

$$
h(t)=\left\{\begin{array}{lll}
K & \text { if } & 0<t \leq t_{1} \\
0 & \text { if } & t_{1}<t \leq t_{1}+t_{2}=\tau
\end{array} .\right.
$$

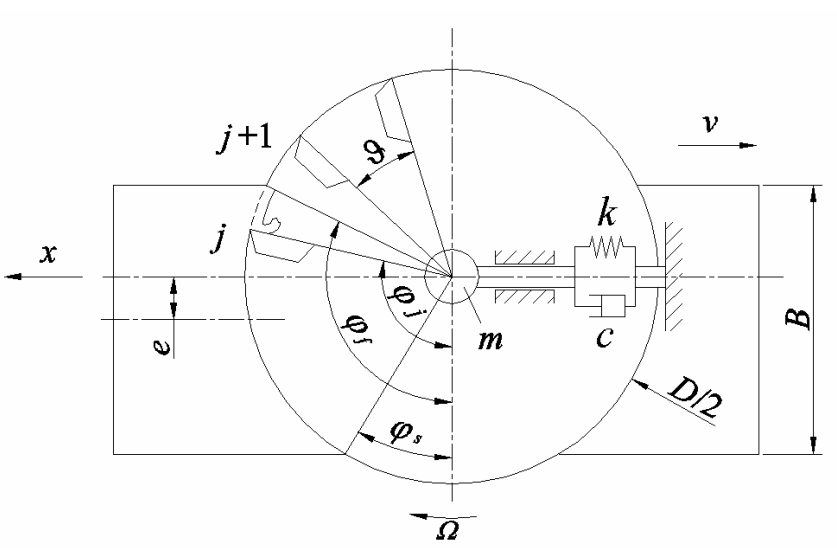

Figure 1. Mechanical model of milling

Putting (2) into (1), the equation of motion reads the form

$$
\ddot{x}(t)+2 \zeta \omega_{n} \dot{x}(t)+\omega_{n}{ }^{2} x(t)=\frac{1}{m} w h(t)(x(t-\tau)-x(t)) .
$$

This is a retarded differential equation with time periodic coefficient. The stability of the trivial solution of equation (4) (i.e. the stability of stationary periodic motion of the tool) can not be analyzed in closed form. The time-periodic delay system can either be approximated by a series of autonomous DDEs, or by a series of time-periodic ordinary differential equations. We choose the latter way by approximating the delayed term as follows

$$
x(t-\tau) \approx \int_{-\infty}^{0} x(t+\vartheta) w_{n}(\vartheta) \mathrm{d} \vartheta
$$

where $w_{n}(\vartheta)$ is a special weight function series coming from the product of a polynomial and an exponential expression

$$
w_{n}(\vartheta)=(-1)^{n} \frac{n^{n+1}}{\tau^{n+1} n !} \vartheta^{n} \exp \left(\frac{n \vartheta}{\tau}\right)
$$

The function $w_{n}(\vartheta)$ satisfies the following properties

$$
\int_{-\infty}^{0} w_{n}(\vartheta) \mathrm{d} \vartheta=1, \quad \lim _{n \rightarrow \infty} w_{n}(\vartheta)=f_{\delta}(\vartheta+\tau)
$$

where $f_{\delta}$ is the Dirac distribution. Fargue (1973) proved that (5) converges to $x(t-\tau)$ as $n$ tends to infinity.

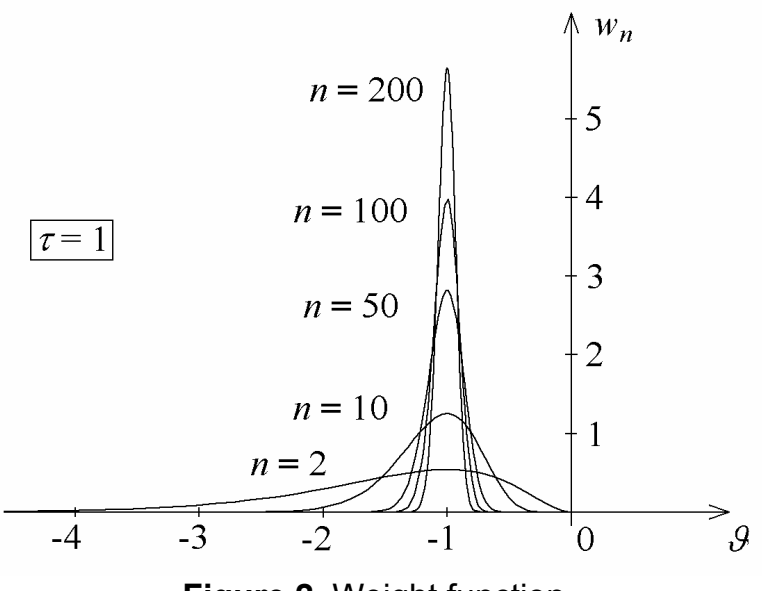

Figure 2. Weight function

Figure 2 shows the weight functions for parameters $n=2$, $10,50,100,200$ and $\tau=1$. It can be seen, that the greater $n$ is, the more correct the approximation is. The convergence looks slow, but without going into the details of the comparison of different approximations used for time-dependent DDEs, the authors find the above approach to be the only reliable and accurate method for stability analysis. This is especially true for systems where the time-dependent parameters have large 
amplitudes relative to the mean values, as it is in case of milling with low number of active teeth.

Apply approximation (5) with a fixed finite $n$ in the equation (4), and introduce the new variables $y_{1}, y_{2}, \ldots, y_{n+3}$ in the following way

$$
\begin{aligned}
& y_{1}(t)=x(t), \\
& y_{2}(t)=\dot{x}(t), \\
& y_{3}(t)=\int_{-\infty}^{0} x(t+\vartheta) w_{n}(\vartheta) \mathrm{d} \vartheta \approx x(t-\tau) .
\end{aligned}
$$

Since

$$
\frac{\mathrm{d}}{\mathrm{d} \vartheta} x(t+\vartheta)=\frac{\mathrm{d}}{\mathrm{d} t} x(t+\vartheta)=\dot{x}(t+\vartheta)
$$

the derivative of $y_{3}(t)$ with respect to the time $t$ can be calculated via integration by parts after the substitution of (6) in (10)

$$
\dot{y}_{3}(t)=-\frac{n}{\tau} y_{3}(t)-\int_{-\infty}^{0} x(t+\vartheta)(-1)^{n} \frac{n^{n+1}}{\tau^{n+1}} \frac{n}{n !} \vartheta^{n-1} \exp \left(\frac{n \vartheta}{\tau}\right) \mathrm{d} \vartheta .
$$

This time, the second term in (12) is defined as the subsequent new variable

$$
y_{4}(t)=\int_{-\infty}^{0} x(t+\vartheta)(-1)^{n} \frac{n^{n+1}}{\tau^{n+1}} \frac{n}{n !} \vartheta^{n-1} \exp \left(\frac{n \vartheta}{\tau}\right) \mathrm{d} \vartheta
$$

where similarly again

$$
\dot{y}_{4}(t)=-\frac{n}{\tau} y_{4}(t)-y_{5}(t)
$$

After the introduction of all the new variables in the same way, and calculating their time derivatives via integration by parts, the degree of $\vartheta$ decreases each time by 1 , till we arrive back to $y_{1}(t)$ at the derivative of $y_{n+3}(t)$

$$
\dot{y}_{n+3}(t)=-\frac{n}{\tau} y_{n+3}(t)-(-1)^{n} \frac{n^{n+1}}{\tau^{n+1}} y_{1}(t) .
$$

The relationship between the new variables defines a finite $(n+3)$ dimensional system of differential equations with a $\tau$ periodic coefficient matrix

$$
\dot{\mathbf{y}}(t)=\mathbf{A}(t) \mathbf{y}(t),
$$

where $\mathbf{y}(t)=\operatorname{col}\left(y_{1} y_{2} \ldots y_{n+3}\right)$ and

$$
\mathbf{A}(t)=\left(\begin{array}{cccccc}
0 & 1 & 0 & 0 & \cdots & 0 \\
-\omega_{n}{ }^{2}-\frac{w}{m} h(t) & -2 \zeta \omega_{n} & \frac{w}{m} h(t) & 0 & \cdots & 0 \\
0 & 0 & \frac{n}{\tau} & -1 & \cdots & 0 \\
\vdots & \vdots & \vdots & \ddots & \ddots & \vdots \\
0 & 0 & 0 & \cdots & \frac{n}{\tau} & -1 \\
(-1)^{n}\left(\frac{n}{\tau}\right)^{n+1} & 0 & 0 & \cdots & & \frac{n}{\tau}
\end{array}\right) .
$$

System (16) is asymptotically stable, if and only if all the characteristic multipliers denoted by $\mu_{j}(j=1,2, \ldots n+3)$ are in modulus less than one, that is, they are inside the unit circle of the complex plane. In general, the characteristic multipliers cannot be calculated in closed form. However, the coefficient matrix $\mathbf{A}(t)$ in (16) is piecewise constant in accordance with the function $h(t)$

$$
\mathbf{A}(t)=\left\{\begin{array}{lll}
\mathbf{A}_{1} & \text { if } & 0<t \leq t_{1} \\
\mathbf{A}_{2} & \text { if } & t_{1}<t \leq t_{1}+t_{2}=\tau
\end{array} .\right.
$$

The characteristic multipliers are the eigenvalues of the principal matrix $\mathbf{C}=\exp \left(\mathbf{A}_{2} t_{2}\right) \exp \left(\mathbf{A}_{1} t_{1}\right)$ (see Farkas, 1994). In critical cases, the relevant multiplier is in modulus 1, i.e. $\left|\mu_{1}\right|=1$, and the frequencies in the corresponding vibration can be found among the imaginary parts

$$
\omega_{k}=\frac{1}{\tau}\left(\operatorname{Im}\left(\ln \left(\mu_{1}\right)\right)+2 k \pi\right), \quad k=0, \pm 1, \pm 2, \ldots
$$

of the characteristic roots $\lambda=(\ln \mu) / \tau$. The lowest $\omega \in[\pi / \tau, 2 \pi / \tau)$ of these frequencies have a central role in identifying the kind of loss of stability discussed in the subsequent section.

\section{STABILITY CHART}

We analyzed the case with the fixed parameters: $m=0.1$ $[\mathrm{kg}], \zeta=0.02, \omega_{n}=3456[\mathrm{~Hz}], K=400\left[\mathrm{~N} / \mathrm{mm}^{2}\right], t_{1} / \tau=0.7$. The horizontal axis is chosen for $z \Omega$, because the tooth pass period $\tau=1 /(z \Omega)$ depends on the number of teeth $z$. The precision of our stability analysis depends on $z \Omega$ for a constant $n$ : the greater $z \Omega$ is, the more accurate the result is. In case of turning with $z \Omega>20[\mathrm{krpm}]$, the $n=200$ approximation gives already an acceptable stability chart with errors under 5\% relative to the exactly known stability limit.

The numerical calculation of the relevant characteristic multipliers shows two kinds of bifurcation phenomena at the stability limits (see Figure 3). At the solid curves, the relevant characteristic multipliers are complex pairs passing through the 
unit circle presenting a secondary Hopf bifurcation, which is similar to the Hopf bifurcation of traditional regenerative chatter theory of turning processes (see Stépán and KalmárNagy, 1997). At the dashed curve, the relevant characteristic multiplier is real, and it passes through the unit circle at -1 resulting a flip (or periodic doubling) bifurcation. This is the same phenomenon experienced by Davies et. al. (2000) for highly interrupted cutting based on a different mechanical model.
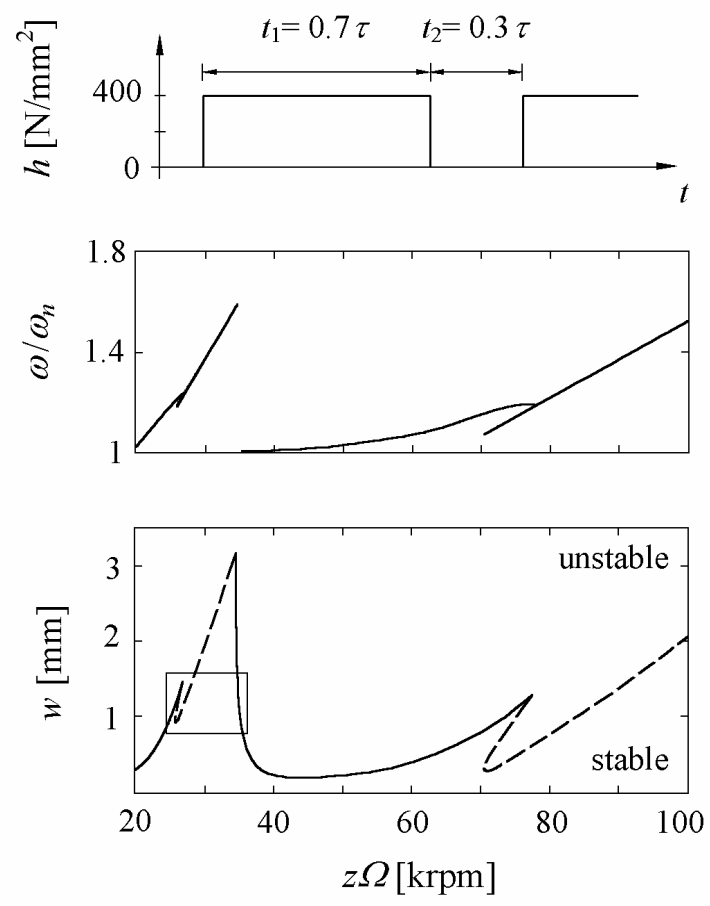

Figure 3. Stability chart

The wandering of the relevant characteristic multipliers is shown in Figure 4. Through the parameter points 1-2-3, the critical characteristic multiplier pair moves into the unit circle presenting a secondary Hopf bifurcation at point 2. Through points 3-4-5, the complex characteristic multiplier pair becomes real, then through 5-6-7, one of them moves out of the unit circle presenting a flip bifurcation at point 6 . Through 7-8-9, the relevant real characteristic multiplier moves back into the unit circle presenting another flip bifurcation at point 8 . Through 9-10-11, the two relevant real characteristic multipliers become a complex conjugate pair again, and decreases in modulus, while through 11-12-13, another complex characteristic multiplier pair moves out of the unit circle on the positive half of the complex plain presenting another secondary Hopf bifurcation at point 12 .

The dashed curve in the chart of Figure 4 presents the parameters where real characteristic multiplier occurs with multiplicity 2 . This curve crosses the intersection of the two kinds of stability limits at $2^{\prime}$ presenting a degenerate (codimension 2) flip bifurcation (see points 1'-2'-3').
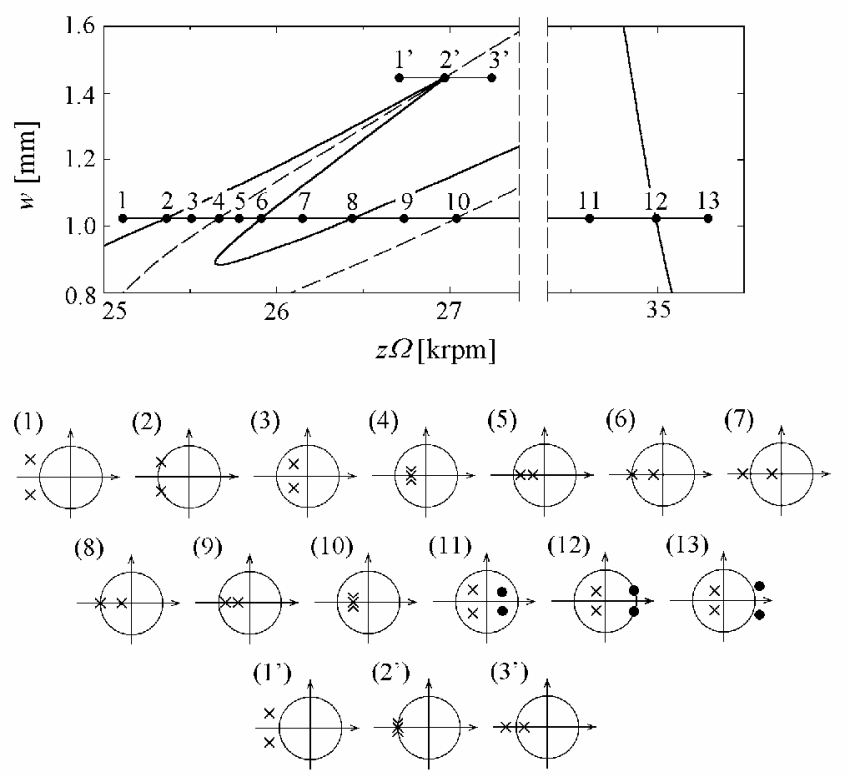

Figure 4. Displacement of relevant characteristic multipliers

In case of flip bifurcation, the frequency of the arising vibration reads

$$
\omega_{k}=\frac{1}{\tau}(\operatorname{Im}(\ln (-1))+2 k \pi)=\frac{1000}{60}(\pi+2 k \pi) z \Omega, \quad k=0,1, \ldots,
$$

that is, it depends linearly on the angular velocity $\Omega$ of the tool, and its lowest value is half of the parametric excitation frequency $z \Omega$. In other words, the time period of the arising vibration is $2 \tau$. The frequency lines are also shown in Figure 3. The first flip-lobe corresponds to $k=0$, the second to $k=1$. In the second case, the tangent of the line is 3 times greater than that of the frequency line above the first flip-lobe.

\section{DISCUSSION}

The stability investigation of the milling process is difficult due to the infinite dimensional phase space caused by the regenerative effect, and due to the parametric excitation caused by the time-varying number of active teeth. Via an approximation of the delayed term, the stability chart in the plane of the technological parameters, and also the essential frequencies of the arising vibrations at the loss of stability are determined. The analysis resulted a new unstable domain in case of high-speed milling associated with period doubling vibrations. If the ratio of time spent cutting to not cutting gets small and the damping is small enough, further new unstable 
domains appear also at lower cutting speeds, similarly to the case of highly interrupted cutting explored by Davies et al. (2000).

\section{ACKNOWLEDGMENTS}

This research was supported by the Hungarian National Science Foundation under grant no. OTKA T030762/99, and the Ministry of Education and Culture grant no. MKM FKFP 0380/97.

\section{REFERENCES}

Balachandran, B., Zhao, M.X., 2000, A Mechanics Based Model for Study of Dynamics of Milling Operations, Meccanica, accepted.

Budak, E., Altintas Y., 1998, Analytical Predection of Chatter Stability in Milling - Part I: General Formulation, Journal of Dynamic Systems, Measurement, and Control 120, pp. 22-30.

Budak, E., Altintas Y., 1998, Analytical Prediction of Chatter Stability in Milling - Part II: Application of the General Formulation to Common Milling Systems, Journal of Dynamic Systems, Measurement, and Control 120, pp. 3136.

Davies, M.A., Pratt, J.R., Dutterer, B., Burns, T.J., 2000, Interrupted Machiening - A Doubling in the Number of Stability Lobes?, The Journal of Manufacturing Science and Engineering, submitted.

Fargue, D., 1973, Réducibilité des systémes héréditaires á des systémes dinamiques, C. R. Acad. Sci. Paris 277B pp. 471473.

Farkas, M., 1994, Periodic Motions, Springer-Verlag, New York.

Hale, J.K., Lunel, S.M.V., 1993, Introduction to Functional Differential Equations, Springer-Verlag, New York.

Minis, I., Yanushevsky, R., 1993, A new theoretical approach for the prediction of machine tool chatter in milling, Journal of Engineering Industry, 115, pp. 1-8.

Moon, F.C., 1998, Dynamics and Chaos in Manufacturing Processes, Wiley, New York.

Seagalman, D.J., Butcher E.A., 2000, Suppression of Regeneratve Chatter via Impedance Modulation, Journal of Vibration and Controll, 6, pp. 243-256.

Shi, H.M., Tobias, S.A., 1984, Theory of finite amplitude machine tool instability, International Journal of Machine Tool Design and Research, 24, pp. 45-69.

Stépán, G., 1989, Retarded dynamical systems, Longman, Harlow.

Stépán, G., 1998, Delay-differential Equation Models for Machine Tool Chatter, in Dynamics and Chaos in Manufacturing Processes, Ed.: Moon, F.C., Wiley, New York, pp. 165-192.

Stépán, G., Kalmár-Nagy, T., 1997, Nonlinear Regenerative Machine Tool Vibration, Proceedings of the $17^{\text {th }}$ Biennial
Conference on Mechanical Vibration and Noise, ASME Design Engineering Technical Conferences, Sacramento, California, September 14-17, 1997.

Tlusty, J., Polacek, A., Danek, C., Spacek, J., 1962, Selbsterregte Schwingungen an Werkzeugmaschinen, VEB Verlag Technik, Berlin.

Tobias, S.A., 1965, Machine Tool Vibration, Blackie, London. 